Article

\title{
Fuzzy Multi-Hypergroups
}

\author{
Sarka Hoskova-Mayerova ${ }^{1, * \mathbb{D}}$, Madeline Al Tahan ${ }^{2} \mathbb{D}$ and Bijan Davvaz ${ }^{3}$ \\ 1 Department of Mathematics and Physics, University of Defence in Brno, Kounicova 65, 66210 Brno-střed, \\ Czech Republic \\ 2 Department of Mathematics, Lebanese International University, Beqaa Valley 1803, Lebanon; \\ madeline.tahan@liu.edu.lb \\ 3 Department of Mathematics, Yazd University, Yazd 89139, Iran; davvaz@yazd.ac.ir \\ * Correspondence: sarka.mayerova@seznam.cz
}

Received: 27 January 2020; Accepted: 12 February 2020; Published: 14 February 2020

check for updates

\begin{abstract}
A fuzzy multiset is a generalization of a fuzzy set. This paper aims to combine the innovative notion of fuzzy multisets and hypergroups. In particular, we use fuzzy multisets to introduce the concept of fuzzy multi-hypergroups as a generalization of fuzzy hypergroups. Different operations on fuzzy multi-hypergroups are defined and discussed and some results known for fuzzy hypergroups are generalized to fuzzy multi-hypergroups.
\end{abstract}

Keywords: hypergroup; multiset; fuzzy multiset; fuzzy multi-hypergroup

MSC: 20N25; 20N20

\section{Introduction}

In 1934, Frederic Marty [1] defined the concept of a hypergroup as a natural generalization of a group. It is well known that the composition of two elements in a group is an element, whereas the composition of two elements in a hypergroup is a non-empty set. The law characterizing such a structure is called the multi-valued operation, or hyperoperation and the theory of the algebraic structures endowed with at least one multi-valued operation is known as the Hyperstructure Theory. Marty's motivation to introduce hypergroups is that the quotient of a group modulo of any subgroup (not necessarily normal) is a hypergroup. Significant progress in the theory of hyperstructures has been made since the 1970s, when its research area was enlarged and different hyperstructures were introduced (e.g., hyperrings, hypermodules, hyperlattices, hyperfields, etc.). Many types of hyperstructures have been used in different contexts, such as automata theory, topology, cryptography and code theory, geometry, graphs and hypergraphs, analysis of the convex systems, finite groups' character theory, theory of fuzzy and rough sets, probability theory, ethnology, and economy [2]. An overview of the most important works and results in the field of hyperstructures up to 1993 is given in the book by Corsini [3], this book was followed in 1994 by the book by Vougiouklis [4]. An overview regarding the applications of hyperstructure theory is given in the book by Corsini and Leoreanu [5]. The book by Davvaz and Leoreanu-Fotea [6] deals with the hyperring theory and applications. A more recent book [7] gives an introduction into fuzzy algebraic hyperstructures.

A set is a well-defined collection of distinct objects, i.e., every element in a set occurs only once. A generalization of the notion of set was introduced by Yager [8]. He introduced the bag (multiset) structure as a set-like object in which repeated elements are significant. He discussed operations on multisets, such as intersection and union, and he showed the usefulness of the new defined structure in relational databases. These new structures have many applications in mathematics and computer science [9]. For example, the prime factorization of a positive integer is a multiset in which its elements are primes (e.g., 90 has the multiset $\{2,3,3,5\}$ ). Moreover, the eigenvalues of a matrix (e.g., the 
$5 \times 5$ lower triangular matrix $\left(a_{i j}\right)$ with $a_{11}=a_{22}=-5, a_{33}=1, a_{44}=a_{55}=0$ has the multiset $\{-5,-5,1,0,0\})$ and roots of a polynomial (e.g., the polynomial $(x+2)^{2}(x-1) x^{3}$ over the field of complex numbers has the multiset $\{-2,-2,1,0,0,0\})$ can be considered as multisets.

As an extension of the classical notion of sets, Zadeh [10] introduced a concept similar to that of a set but whose elements have degrees of membership and he called it fuzzy set. In classical sets, the membership function can take only two values: 0 and 1 . It takes 0 if the element belongs to the set, and 1 if the element does not belong to the set. In fuzzy sets, there is a gradual assessment of the membership of elements in a set which is assigned a number between 0 and 1 (both included). Several applications for fuzzy sets appear in real life. We refer to the papers [11-13]. Yager, in [8], generalized the fuzzy set by introducing the concept of fuzzy multiset (fuzzy bag) and he discussed a calculus for them in [14]. An element of a fuzzy multiset can occur more than once with possibly the same or different membership values. If every element of a fuzzy multiset can occur at most once, we go back to fuzzy sets [15].

In [16], Onasanya and Hoskova-Mayerova defined multi-fuzzy groups and in [17,18], the authors defined fuzzy multi-polygroups and fuzzy multi- $H_{v}$-ideals and studied their properties. Moreover, Davvaz in [19] discussed various properties of fuzzy hypergroups. Our paper generalizes the work in $[16,17,19]$ to combine hypergroups and fuzzy multisets. More precisely, it is concerned with fuzzy multi-hypergroups and constructed as follows: After the Introduction, Section 2 presents some preliminary definitions and results related to fuzzy multisets and hypergroups that are used throughout the paper. Section 3 introduces, for the first time, fuzzy multi-hypergroups as a generalization of fuzzy hypergroups and studies its properties. Finally, Section 4 defines some operations (e.g., intersection, selection, product, etc.) on fuzzy multi-hypergroups and discusses them.

\section{Preliminaries}

In this section, we present some basic definitions and results related to both fuzzy multisets and hypergroups $[20,21]$ that are used throughout the paper.

\subsection{Fuzzy Multisets}

A multiset is a collection of objects that can be repeated. Yager in his paper [8] introduced, under the name $b a g$, a structure similar to a set in which repeated elements are allowed. He studied some operations on bags, such as intersection, union, and addition. Moreover, he introduced the operation of selecting elements from a bag based upon their membership in a set. Furthermore, he suggested a definition for fuzzy multisets (fuzzy bags). A multiset (bag) $M$ is characterized by a count function $C_{M}: X \rightarrow \mathbb{N}$, where $\mathbb{N}$ is the set of natural numbers.

In a multiset, and unlike a set, the multiple occurrences for each of its elements is allowed and it is called multiplicity. For example, the multiset $\{\alpha, \beta\}$ contains two elements $\alpha$ and $\beta$, each having multiplicity 1 , whereas the multiset $\{\alpha, \beta, \beta\}$ contains two elements $\alpha$ having multiplicity 1 and $\beta$ having multiplicity 2 . The two multisets $\{\alpha, \beta, \beta\}$ and $\{\alpha, \beta\}$ are not equal although they are considered equal as sets.

Definition $1([17,22])$. Let $X$ be a set. A multiset $M$ drawn from $X$ is represented by a function $C_{M}: X \rightarrow$ $\{0,1,2, \ldots\}$. For each $x \in X, C_{M}(x)$ denotes the number of occurrences of $x$ in $M$.

Assume $X=\left\{a_{1}, \ldots, a_{k}\right\}$. For a multiset $M$ on $X$ with count function $C_{M}$, the following two equivalent expressions are used:

$$
M=\left\{a_{1} / n_{1}, \ldots, a_{k} / n_{k}\right\}
$$

and

$$
M=\{\underbrace{a_{1}, \ldots, a_{1}}_{n_{1}}, \ldots, \underbrace{a_{k}, \ldots, a_{k}}_{n_{k}}\} .
$$


Here, $x_{i}$ has multiplicity $n_{i}$ for all $i=1, \ldots, k$, or equivalently, $C_{M}\left(x_{i}\right)=n_{i}$ for all $i=1, \ldots, k$.

One potential useful application for the theory of multisets lies in the field of relational databases [8].

Example 1. Consider a class of eight students and a relation of students over the scheme (student name, grade) given as follows:

$R=\{($ Sam, 90$),($ Nader, 85),$($ Mady, 90), $($ Tala, 60$),(J o e, 70),(Z i a d, 95),($ Lune, 85), (Bella, 60) $\}$. Assume we are interested in finding the grades of students in this class. We can take the projection of $R$ on grades and get:

$$
\text { Proj }_{\text {grade }}=\{60,70,85,90,95\} .
$$

The set Projgrade does not give the set of grades of all students in the class but it gives the set of different grades of the students. If we need the set of grades of all students in the class then we need to consider the following multiset $M$ :

$$
M=\{60,60,70,85,85,90,90,95\}=\{60 / 2,70 / 1,85 / 2,90 / 2,95 / 1\} .
$$

Or equivalently, we can say that $M$ is a multiset with count function $C_{M}$ defined as follows: $C_{M}(60)=$ $C_{M}(85)=C_{M}(90)=2$ and $C_{M}(70)=C_{M}(95)=1$. The notation used here for the multiset $M$ is the same as that used by Yager in his pioneering paper [8] about multisets.

Definition 2 ([23]). Let $X$ be a non-empty set. A fuzzy multiset A drawn from $X$ is represented by a function $C M_{A}: X \rightarrow Q$, where $Q$ is the set of all multisets drawn from the unit interval $[0,1]$.

In the above definition, the value $C M_{A}(x)$ is a multiset drawn from $[0,1]$. For each $x \in X$, the membership sequence is defined as the decreasingly ordered sequence of elements in $C M_{A}(x)$ and it is denoted by:

$$
\left\{\mu_{A}^{1}(x), \mu_{A}^{2}(x), \ldots, \mu_{A}^{p}(x)\right\}: \mu_{A}^{1}(x) \geq \mu_{A}^{2}(x) \geq \ldots \geq \mu_{A}^{p}(x) .
$$

Fuzzy sets introduced by Zadeh [10] can be considered as a special case of fuzzy multisets by setting $p=1$ so that $C M_{A}(x)=\mu_{A}^{1}(x)$.

Example 2. Let $X=\{0,1,2,3\}$. Then $A=\{(0.5,0.5,0.3,0.1,0.1) / 1,(0.65,0.2,0.2,0.2) / 3\}$ is a fuzzy multiset of $X$ with fuzzy count function $C M_{A}$. Or equivalently, we can write it as:

$$
C M_{A}(0)=C M_{A}(2)=0, C M_{A}(1)=(0.5,0.5,0.3,0.1,0.1), C M_{A}(3)=(0.65,0.2,0.2,0.2) .
$$

Let $A, B$ be fuzzy multisets of $X$ with fuzzy count functions $C M_{A}, C M_{B}$ respectively. Then $L(x ; A)=\max \left\{j: \mu_{A}^{j}(x) \neq 0\right\}$ and $L(x)=L(x ; A, B)=\max \{L(x ; A), L(x ; B)\}$. When we define an operation between two fuzzy multisets, the length of their membership sequences should be set as equal. In case two membership sequences have different lengths then the shorter sequence is extended with zeros. As an illustration, we consider the following example.

Example 3. Let $X=\{0,1,2,3\}$ and define the fuzzy multisets $A, B$ of $X$ as follows:

$$
\begin{aligned}
& A=\{(0.4,0.2) / 0,(0.5,0.3,0.1) / 1,(0.65,0.2,0.2,0.2) / 3\}, \\
& B=\{(0.5,0.3,0.1,0.1) / 1,(0.5,0.1) / 2,(0.65,0.2,0.2) / 3\} .
\end{aligned}
$$

In order to define any operations on $A, B$, we can rewrite $A, B$ as follows:

$$
\begin{aligned}
& A=\{(0.4,0.2) / 0,(0.5,0.3,0.1,0) / 1,(0,0) / 2,(0.65,0.2,0.2,0.2) / 3\}, \\
& B=\{(0,0) / 0,(0.5,0.3,0.1,0.1) / 1,(0.5,0.1) / 2,(0.65,0.2,0.2,0) / 3\} .
\end{aligned}
$$


Definition 3 ([24]). Let $X$ be a set and A,B be fuzzy multisets of $X$. Then

1. $A \subseteq B$ if and only if $C M_{A}(x) \leq C M_{B}(x)$ for all $x \in X$. i.e., $\mu_{A}^{j}(x) \leq \mu_{B}^{j}(x), j=1, \ldots, L(x)$ for all $x \in X$,

2. $A=B$ if and only if $C M_{A}(x)=C M_{B}(x)$ for all $x \in X$. i.e., $\mu_{A}^{j}(x)=\mu_{B}^{j}(x), j=1, \ldots, L(x)$ for all $x \in X$,

3. $A \cap B$ is defined by $\mu_{A \cap B}^{j}(x)=\mu_{A}^{j}(x) \wedge \mu_{B}^{j}(x), j=1, \ldots, L(x)$ for all $x \in X$,

4. $A \cup B$ is defined by $\mu_{A \cap B}^{j}(x)=\mu_{A}^{j}(x) \vee \mu_{B}^{j}(x), j=1, \ldots, L(x)$ for all $x \in X$.

Example 4. Let $X=\{0,1,2,3\}$ and define the fuzzy multisets $A, B, C$ of $X$ as follows:

$$
\begin{gathered}
A=\{(0.5,0.3,0.1,0.1) / 1,(0.65,0.2,0.2,0.2) / 3\}, \\
B=\{(0.5,0.3,0.1,0.1) / 1,(0.5,0.1) / 2,(0.65,0.2,0.2,0.2) / 3\}, \\
C=\{(0.5,0.3) / 0,(0.5,0.1) / 2,(0.35,0.3,0.1) / 3\} .
\end{gathered}
$$

Then it is clear that:

1. $A \subseteq B$ and $A \neq B$,

2. $A \cap C=\{(0.35,0.2,0.1) / 3\}$, and

3. $A \cup C=\{(0.5,0.3) / 0,(0.5,0.3,0.1,0.1) / 1,(0.5,0.1) / 2,(0.65,0.3,0.2,0.2) / 3\}$.

Definition 4 ([25]). Let $X, Y$ be non-empty sets, $f: X \rightarrow Y$ be a mapping, and $A, B$ be fuzzy multisets of $X, Y$ respectively. Then

1. The image of $A$ under $f$ is denoted by $f(A)$ with fuzzy count function $C M_{f(A)}$ defined as follows: For all $y \in Y$,

$$
C M_{f(A)}(y)= \begin{cases}\bigvee_{f(x)=y} C M_{A}(x) & \text { if } f^{-1}(y) \neq \varnothing \\ 0 & \text { otherwise. }\end{cases}
$$

2. The inverse image of $B$ under $f$ is denoted by $f^{-1}(B)$ with fuzzy count function $C M_{f^{-1}(B)}$ defined as: $C M_{f^{-1}(B)}(x)=C M_{B}(f(x))$ for all $x \in X$.

\subsection{Hypergroups}

Let $H$ be a non-empty set and $\mathcal{P}^{*}(H)$ be the family of all non-empty subsets of $H$. Then, a mapping $\circ: H \times H \rightarrow \mathcal{P}^{*}(H)$ is called a binary hyperoperation on $H$ and $(H, \circ)$ is called a hypergroupoid.

In the above definition, if $A$ and $B$ are two non-empty subsets of $H$ and $x \in H$, then we define:

$$
A \circ B=\bigcup_{\substack{a \in A \\ b \in B}} a \circ b, x \circ A=\{x\} \circ A \text { and } A \circ x=A \circ\{x\} .
$$

A hypergroupoid $(H, \circ)$ is called a semihypergroup if "०" is associative, i.e., if $x \circ(y \circ z)=(x \circ y) \circ z$ for all $x, y, z \in H$ and is called a quasihypergroup if the reproduction axiom is satisfied, i.e., if $x \circ H=$ $H=H \circ x$ for all $x \in H$. The couple $(H, \circ)$ is called a hypergroup if it is a semihypergroup and a quasihypergroup. A hypergroup $(H, \circ)$ is called commutative if $x \circ y=y \circ x$ for all $x, y \in H$. A subset $S$ of a hypergroup $(H, \circ)$ is called a subhypergroup of $H$ if $(S, \circ)$ is a hypergroup. To prove that $S$ is a subhypergroup of $H$, it suffices to show that the reproduction axiom is satisfied for $S$.

Example 5. In the finite hypergroup, we can represent the hyperoperation by the Cayley square table in the same way as for the finite group. The only difference is that various places in the table may contain several elements instead of a single element, i.e.,

\begin{tabular}{|c||c|c|c|}
\hline$*$ & $e$ & $a$ & $b$ \\
\hline$e$ & $\{e\}$ & $\{a, b\}$ & $\{a, b\}$ \\
\hline$a$ & $\{a\}$ & $\{e, b\}$ & $\{e, b\}$ \\
\hline$b$ & $\{b\}$ & $\{e, a\}$ & $\{e, a\}$ \\
\hline
\end{tabular}


is a hypergroup.

Definition $5([17,26])$. Let $\left(H_{1}, o_{1}\right)$ and $\left(H_{2}, o_{2}\right)$ be hypergroups and $f: H_{1} \rightarrow H_{2}$ be a mapping. Then $f$ is:

1. a homomorphism if $f\left(x \circ_{1} y\right) \subseteq f(x) \circ_{2} f(y)$ for all $x, y \in H_{1}$;

2. a strong homomorphism if $f\left(x \circ_{1} y\right)=f(x) \circ_{2} f(y)$ for all $x, y \in H_{1}$;

3. an isomorphism if $f$ is a bijective strong homomorphism.

Example 6. Let $H$ be any non-empty set and define o on $H$ as follows:

$$
x \circ y=H \text { for all } x, y \in H \text {. }
$$

Then $(H, \circ)$ is a hypergroup known as total hypergroup.

Example 7. Let $H$ be any non-empty set and define $\circ$ on $H$ as follows:

$$
x \circ y=\{x, y\} \text { for all } x, y \in H .
$$

Then $(H, \circ)$ is a hypergroup known as biset hypergroup.

Example 8. Let $\mathbb{Z}$ be the set of integers and define $\star$ on $\mathbb{Z}$ as follows: For all $x, y \in \mathbb{Z}$,

$$
x \star y= \begin{cases}2 \mathbb{Z} & \text { if } x, y \text { have same parity } \\ 2 \mathbb{Z}+1 & \text { otherwise. }\end{cases}
$$

Then $(\mathbb{Z}, \star)$ is a commutative hypergroup.

For more examples and details about hypergroups, we refer to the books $[3-6,26,27]$ and to the papers $[20,21,28-32]$.

\section{Construction of Fuzzy Multi-Hypergroups}

Inspired by the definition of the multi-fuzzy group [25] and the fuzzy multi-polygroup [17], we introduce the concept of the fuzzy multi-hypergroup. Further, we investigate their properties. It is well known that groups and polygroups [26] are considered as special cases of hypergroups. Hence, the results in this section can be considered as more general than that in $[17,25]$.

Definition 6 ([7]). Let $(H, \circ)$ be a hypergroup and $\mu$ be a fuzzy subset of $H$. Then $\mu$ is called a fuzzy subhypergroup of $H$ if for all $x, y \in H$, the following conditions hold.

1. $\mu(x) \wedge \mu(y) \leq \inf _{z \in x \circ y} \mu(z)$;

2. for every $x, a \in H$ there exists $y \in H$ such that $x \in a \circ y$ and $\mu(x) \wedge \mu(a) \leq \mu(y)$;

3. for every $x, a \in H$ there exists $z \in H$ such that $x \in z \circ a$ and $\mu(x) \wedge \mu(a) \leq \mu(z)$.

Definition 7. Let $(H, \circ)$ be a hypergroup. A fuzzy multiset A over $H$ is a fuzzy multi-hypergroup of $H$ if for all $x, y \in H$, the following conditions hold.

1. $C M_{A}(x) \wedge C M_{A}(y) \leq \inf _{z \in x \circ y} C M_{A}(z)$ (or equivalently, $C M_{A}(x) \wedge C M_{A}(y) \leq C M_{A}(z)$ for all $z \in x \circ y)$;

2. for every $x, a \in H$ there exists $y \in H$ such that $x \in a \circ y$ and $C M_{A}(x) \wedge C M_{A}(a) \leq C M_{A}(y)$;

3. for every $x, a \in H$ there exists $z \in H$ such that $x \in z \circ$ a and $C M_{A}(x) \wedge C M_{A}(a) \leq C M_{A}(z)$.

Remark 1. It is clear, using Definitions 6 and 7 , that if $(H, \circ)$ is a hypergroup and $\mu$ is a fuzzy subhypergroup of $H$ then $\mu$ is a fuzzy multi-hypergroup of $H$. Hence, the results of fuzzy subhypergroups are considered a special case of our work. 
Remark 2. Let $A$ be a fuzzy multiset over a commutative hypergroup $(H, \circ)$. Then conditions 2. and 3. of Definition 7 are equivalent. Hence, to show that $A$ is a fuzzy multi-hypergroup of $H$, it suffices to show that either conditions 1. and 2. of Definition 7 are valid or conditions 1. and 3. of Definition 7 are valid.

We present different examples on fuzzy multi-hypergroups.

Example 9. Let $(H, \circ)$ be the hypergroup defined by the following table:

\begin{tabular}{|c|c|c|}
\hline$\circ$ & $a$ & $b$ \\
\hline$a$ & $H$ & $a$ \\
\hline$b$ & $a$ & $b$ \\
\hline
\end{tabular}

It is clear that $A=\{(0.2,0.1) / a,(0.5,0.4,0.4) / b\}$ is a fuzzy multi-hypergroup of $H$.

Example 10. Let $\left(H_{1}, \circ_{1}\right)$ be the hypergroup defined by the following table:

\begin{tabular}{|c|c|c|c|}
\hline$\circ_{1}$ & 0 & 1 & 2 \\
\hline 0 & $\{0,1\}$ & $\{0,1\}$ & $H_{1}$ \\
\hline 1 & $\{0,1\}$ & $\{0,1\}$ & $H_{1}$ \\
\hline 2 & $H_{1}$ & $H_{1}$ & 2 \\
\hline
\end{tabular}

It is clear that $A=\{(0.2,0.1) / 0,(0.2,0.1) / 1,(0.5,0.4,0.4) / 2\}$ is a fuzzy multi-hypergroup of $H_{1}$.

Example 11. Let $(\mathbb{Z}, \star)$ be the hypergroup defined in Example 8. It is clear that $A$, with the fuzzy count function $C M_{A}$, is a fuzzy multi-hypergroup of $\mathbb{Z}$. Where

$$
C M_{A}(x)= \begin{cases}(0.7,0.5,0.5) & \text { if } x \text { is an even integer } \\ (0.7,0.3,0.2) & \text { otherwise. }\end{cases}
$$

Proposition 1. Let $(H, \circ)$ be a hypergroup and $A$ be a fuzzy multi-hypergroup of $H$. Then the following assertions are true.

1. $C M_{A}(z) \geq C M_{A}\left(x_{1}\right) \wedge \ldots \wedge C M_{A}\left(x_{n}\right)$ for all $z \in x_{1} \circ \ldots \circ x_{n}$ and $n \geq 2$;

2. $C M_{A}(z) \geq C M_{A}(x)$ for all $z \in x^{n}$.

\section{Proof.}

- Proof of 1. By mathematical induction on the value of $n, C M_{A}(z) \geq C M_{A}\left(x_{1}\right) \wedge \ldots \wedge C M_{A}\left(x_{n}\right)$ for all $z \in x_{1} \circ \ldots \circ x_{n}$ is true for $n=2$. Assume that $C M_{A}(z) \geq C M_{A}\left(x_{1}\right) \wedge \ldots \wedge C M_{A}\left(x_{n}\right)$ for all $z \in x_{1} \circ \ldots \circ x_{n}$ and let $z^{\prime} \in x_{1} \circ \ldots \circ x_{n} \circ x_{n+1}$. Then there exists $x \in x_{1} \circ \ldots \circ x_{n}$ such that $z^{\prime} \in$ $x \circ x_{n+1}$. Having $A$ a fuzzy multi-hypergroup implies that $C M_{A}\left(z^{\prime}\right) \geq C M_{A}(x) \wedge C M_{A}\left(x_{n+1}\right)$. And using our assumption that $C M_{A}(x) \geq C M_{A}\left(x_{1}\right) \wedge \ldots \wedge C M_{A}\left(x_{n}\right)$ implies that our statement is true for $n+1$.

- Proof of 2 . The proof follows from 1 . by setting $x_{i}=x$ for all $i=1, \ldots, n$.

Example 12. Let $(H, \circ)$ be any hypergroup and $a \in H$ be a fixed element. We define a fuzzy multiset $A$ of $H$ with fuzzy count function $C M_{A}$ as $C M_{A}(x)=C M_{A}(a)$ for all $x \in H$. Then $A$ is a fuzzy multi-hypergroup of $H$ (the constant fuzzy multi-hypergroup).

Remark 3. Let $(H, \circ)$ be a hypergroup. Then we can define at least one fuzzy multi-hypergroup of $H$, which is mainly the one that is described in Example 12. 
Proposition 2. Let $(H, \circ)$ be the biset hypergroup and $A$ be a fuzzy multiset of $H$. Then $A$ is a fuzzy multi-hypergroup of $H$.

Proof. Let $A$ be a fuzzy multiset of $H$. Since $(H, \circ)$ is a commutative hypergroup, it suffices to show that conditions 1. and 2. of Definition 7 are satisfied. (See Remark 2). For condition 1., let $x, y \in H$ and $z \in x \circ y=\{x, y\}$. It is clear that $C M_{A}(z) \geq C M_{A}(x) \wedge C M_{A}(y)$. For condition 2., let $a, x \in H$. Then there exists $y=x \in H$ such that $x \in a \circ y$ and $C M_{A}(y)=C M_{A}(x) \geq C M_{A}(a) \wedge C M_{A}(x)$. Therefore, $A$ is a fuzzy multi-hypergroup of $H$.

Proposition 3. Let $(H, \circ)$ be the total hypergroup and $A$ be a fuzzy multiset of $H$. Then $A$ is a fuzzy multi-hypergroup of $H$ if and only if $A$ is the fuzzy multiset described in Example 12.

Proof. If $A$ is the fuzzy multiset described in Example 12 then it is clear that $A$ is a fuzzy multi-hypergroup of $H$. Let $A$ be a fuzzy multi-hypergroup of $H$ and $a \in H$. For all $x \in H$, we have $x \in a \circ a=H$ and $a \in x \circ x$. The latter and having $A$ a fuzzy multi-hypergroup of $H$ implies that $C M_{A}(x) \geq C M_{A}(a)$ and $C M_{A}(a) \geq C M_{A}(x)$. Thus, $C M_{A}(x)=C M_{A}(a)$ for all $x \in H$.

Notation 1. Let $(H, 0)$ be a hypergroup, $A$ be a fuzzy multiset of $H$ and $C M_{A}(x)=$ $\left(\mu_{A}^{1}(x), \mu_{A}^{2}(x), \ldots, \mu_{A}^{p}(x)\right)$. We say that $C M_{A}(x)>0$ if $\mu_{A}^{1}(x)>0$.

Definition 8. Let $(H, \circ)$ be a hypergroup and $A$ be a fuzzy multiset of $H$. Then $A_{\star}=\left\{x \in X: C M_{A}(x)>0\right\}$.

Proposition 4. Let $(H, \circ)$ be a hypergroup and $A$ be a fuzzy multi-hypergroup of $H$. Then $A_{\star}$ is either the empty set or a subhypergroup of $H$.

Proof. Let $a \in A_{\star} \neq \varnothing$. We need to show that the reproduction axiom is satisfied for $A_{\star}$. We show that $a \circ A_{\star}=A_{\star}$ and $A_{\star} \circ a=A_{\star}$ is done similarly. For all $x \in A_{\star}$ and $z \in a \circ x$, we have $C M_{A}(z) \geq C M_{A}(a) \wedge C M_{A}(x)>0$. The latter implies that $z \in A_{\star}$ and hence, $A_{\star} \circ a \subseteq A_{\star}$. Moreover, for all $x \in A_{\star}$, Condition 2. of Definition 7 implies that there exist $y \in H$ such that $x \in a \circ y$ and $C M_{A}(y) \geq C M_{A}(x) \wedge C M_{A}(a)>0$. The latter implies that $y \in A_{\star}$ and $x \in a \circ A_{\star}$. Thus, $A_{\star} \subseteq a \circ A_{\star}$.

Definition 9. Let $(H, \circ)$ be a hypergroup and $A, B$ be fuzzy multisets of $H$. Then $A \circ B$ is defined by the following fuzzy count function.

$$
C M_{A \circ B}(x)=\vee\left\{C M_{A}(y) \wedge C M_{B}(z): x \in y \circ z\right\} .
$$

Theorem 1. Let $(H, O)$ be a hypergroup and $A$ be a fuzzy multiset of $H$. If $A$ is a fuzzy multi-hypergroup of $H$ then $A \circ A=A$.

Proof. Let $z \in H$. Then $C M_{A}(z) \geq C M_{A}(x) \wedge C M_{A}(y)$ for all $z \in x \circ y$. The latter implies that $C M_{A}(z) \geq \vee\left\{C M_{A}(x) \wedge C M_{B}(y): z \in x \circ y\right\} \geq C M_{A \circ A}(z)$. Thus, $A \circ A \subseteq A$. Having $(H, \circ) \mathrm{a}$ hypergroup and $A$ a fuzzy multi-hypergroup of $H$ implies that for every $x \in H$ there exist $y \in H$ such that $x \in x \circ y$ and $C M_{A}(y) \geq C M_{A}(x)$. Moreover, we have $C M_{A \circ A}(x)=\vee\left\{C M_{A}(y) \wedge C M_{B}(z): x \in\right.$ $y \circ z\} \geq C M_{A}(x) \wedge C M_{A}(y)=C M_{A}(x)$. Thus, $A \subseteq A \circ A$.

Notation 2. Let $(H, \circ)$ be a hypergroup, $A$ be a fuzzy multiset of $H$ and $C M_{A}(x)=$ $\left(\mu_{A}^{1}(x), \mu_{A}^{2}(x), \ldots, \mu_{A}^{p}(x)\right)$. We say that $C M_{A}(x) \geq\left(t_{1}, \ldots, t_{k}\right)$ if $p \geq k$ and $\mu_{A}^{i}(x) \geq t_{i}$ for all $i=1, \ldots, k$. If $C M_{A}(x) \ngtr\left(t_{1}, \ldots, t_{k}\right)$ and $\left(t_{1}, \ldots, t_{k}\right) \ngtr C M_{A}(x)$ then we say that $C M_{A}(x)$ and $\left(t_{1}, \ldots, t_{k}\right)$ are not comparable. 
In [19], Davvaz studied fuzzy hypergroups and proved some important results about them related to level subhypergroups of fuzzy hypergroups. In what follows, we do suitable changes to extend the results of [19] to fuzzy multi-hypergroups.

Theorem 2. Let $(H, \circ)$ be a hypergroup, A a fuzzy multiset of $H$ with fuzzy count function $C M$ and $t=$ $\left(t_{1}, \ldots, t_{k}\right)$ where $t_{i} \in[0,1]$ for $i=1, \ldots, k$ and $t_{1} \geq t_{2} \geq \ldots \geq t_{k}$. Then $A$ is a fuzzy multi-hypergroup of $H$ if and only if $C M_{t}$ is either the empty set or a subhypergroup of $H$.

Proof. Let $C M_{t}$ be a subhypergroup of $H$ and $x, y \in H$. By setting $t_{0}=C M(x) \wedge C M(y)$, we get that $x, y \in C M_{t_{0}}$. Having $C M_{t_{0}}$ a subhypergroup of $H$ implies that for all $z \in x \circ y, C M(z) \geq$ $t_{0}=C M(x) \wedge C M(y)$. We prove condition 2. of Definition 7 and condition 3. is done similarly. Let $a, x \in H$ and $t_{0}=C M(x) \wedge C M(a)$. Then $a, x \in C M_{t_{0}}$. Having $C M_{t_{0}}$ a subhypergroup of $H$ implies that $a \circ C M_{t_{0}}=C M_{t_{0}}$. The latter implies that there exist $y \in C M_{t_{0}}$ such that $x \in a \circ y$. Thus, $C M(y) \geq t_{0}=C M(x) \wedge C M(y)$.

Conversely, let $A$ be a fuzzy multi-hypergroup of $H$ and $C M_{t} \neq \varnothing$. We need to show that $C M_{t}=a \circ C M_{t}=C M_{t} \circ a$ for all $a \in C M_{t}$. We prove that $C M_{t}=a \circ C M_{t}$ and $C M_{t}=C M_{t} \circ a$ is done similarly. Let $x \in C M_{t}$. Then $C M(z) \geq C M(x) \wedge C M(a) \geq t$ for all $z \in a \circ x$. The latter implies that $z \in C M_{t}$. Thus, $a \circ C M_{t} \subseteq C M_{t}$. Let $x \in C M_{t}$. Since $A$ is a fuzzy multi-hypergroup of $H$, it follows that there exist $y \in H$ such that $x \in a \circ y$ and $C M(y) \geq C M(x) \wedge C M(a) \geq t$. The latter implies that $y \in C M_{t}$ and hence, $C M_{t} \subseteq a \circ C M_{t}$.

Proposition 5. Let $(H, \circ)$ be a hypergroup, A a fuzzy multiset of $H$ with fuzzy count function $C M$ and $t=\left(t_{1}, \ldots, t_{k}\right), s=\left(s_{1}, \ldots, s_{n}\right)$ where $t_{i}, s_{i} \in[0,1]$ for $i=1, \ldots, \max (k, n)$ and $t_{1} \geq t_{2} \geq \ldots \geq t_{k}$, $s_{1} \geq s_{2} \geq \ldots \geq s_{n}$. If $t<s$ and $C M_{t}=C M_{s}$ then there exist no $x \in H$ such that $t \leq C M(x)<s$.

Proof. Let $C M_{t}=C M_{s}$ and suppose that there exist $x \in H$ such that $t \leq C M(x)<s$. Then $x \in C M_{t}$ and $x \notin C M_{S}$ which contradicts the given.

Proposition 6. Let $(H, \circ)$ be a hypergroup and $S$ be a subhypergroup of $H$. Then $S=C M_{t}$ for some $t=\left(t_{1}, \ldots, t_{k}\right)$ where $t_{i} \in[0,1]$ for $i=1, \ldots, k, t_{1} \neq 0$, and $t_{1} \geq t_{2} \geq \ldots \geq t_{k}$.

Proof. Let $t=\left(t_{1}, \ldots, t_{k}\right)$ where $t_{i} \in[0,1]$ for $i=1, \ldots, k$ and define the fuzzy multiset $A$ of $H$ as follows:

$$
C M(x)= \begin{cases}t & \text { if } x \in S \\ 0 & \text { otherwise. }\end{cases}
$$

It is clear that $S=C M_{t}$. We still need to prove that $C M$ is a fuzzy multi-hypergroup of $H$. Using Theorem 2, it suffices to show that $C M_{\alpha} \neq \varnothing$ is a subhypergroup of $H$ for all $\alpha=\left(a_{1}, \ldots, a_{s}\right)$ with $a_{i} \in[0,1]$ for $i=1, \ldots$, s. One can easily see that

$$
C M_{\alpha}= \begin{cases}H & \text { if } \alpha=0 \\ S & \text { if } 0<\alpha \leq t \\ \varnothing & \text { if }(\alpha>t) \text { or }(\alpha \text { and } t \text { are not comparable }) .\end{cases}
$$

Thus, $C M_{\alpha}$ is either the empty set or a subhypergroup of $H$.

\section{Operations on Fuzzy Multi-Hypergroups}

In this section, we define some operations on fuzzy multi-hypergroups, study them, and present some examples. 
Proposition 7. Let $(H, O)$ be a hypergroup and $A, B$ be fuzzy multisets of $H$. If $A$ and $B$ are fuzzy multi-hypergroups of $H$ and one of them is the constant fuzzy multi-hypergroup then $A \cap B$ is a fuzzy multi-hypergroup of $H$.

Proof. We prove conditions of Definition 7 are satisfied for $A \cap B$. (1) Let $x, y \in H$ and $z \in$ $x \circ y$. Then $C M_{A \cap B}(z)=C M_{A}(z) \wedge C M_{B}(z)$. Having $A, B$ fuzzy multi-hypergroups of $H$ implies that $C M_{A}(z) \geq C M_{A}(x) \wedge C M_{A}(y)$ and $C M_{B}(z) \geq C M_{B}(x) \wedge C M_{B}(y)$. The latter implies that $C M_{A \cap B}(z) \geq C M_{A}(x) \wedge C M_{A}(y) \wedge C M_{B}(x) \wedge C M_{B}(y)=C M_{A \cap B}(x) \wedge C M_{A \cap B}(y)$. (2) Without loss of generality, let $B$ be the constant fuzzy multiset of $H$ with $C M_{B}(x)=\alpha$ for all $x \in H$. Let $a, x \in H$. Then there exist $y \in H$ such that $x \in a \circ y$ and $C M_{A}(y) \geq C M_{A}(x) \wedge C M_{A}(a)$. The latter implies that $C M_{A \cap B}(y)=C M_{A}(y) \wedge \alpha \geq C M_{A}(x) \wedge C M_{A}(a) \wedge \alpha=\left(C M_{A}(x) \wedge \alpha\right) \wedge\left(C M_{A}(a) \wedge \alpha\right)=$ $C M_{A \cap B}(x) \wedge C M_{A \cap B}(a)$. (3) is done in a similar way to (2).

Example 13. Let $(H, \circ)$ be the hypergroup defined in Example 9 and $A, B$ be fuzzy multisets of $H$ defined as:

$$
A=\{(0.2,0.1) / a,(0.5,0.4,0.4) / b\}, B=\{(0.7,0.05,0.05) / a,(0.7,0.05,0.05) / b\} .
$$

Since $A$ is a fuzzy multi-hypergroup of $H$ and $B$ is a constant fuzzy multi-hypergroup of $H$, it follows that $A \cap B=\{(0.2,0.05) / a,(0.5,0.05,0.05) / b\}$ is a fuzzy multi-hypergroup of $H$.

Definition 10. Let $H$ be any set and $A, B$ be fuzzy multisets of $H$ with fuzzy count functions $C M_{A}, C M_{B}$ respectively. Then the fuzzy multiset $A \biguplus B$ is given as follows: For all $x \in H$,

$$
C M(x)=\frac{C M_{A}(x)+C M_{B}(x)}{2} .
$$

Example 14. Let $H=\{a, b\}, A, B$ be fuzzy multisets of $H$ given as :

$$
A=\{(0.8,0.4,0.4) / a,(0.1,0.1) / b\}, B=\{(0.6,0.4,0.4,0.4) / a,(0.3,0.1,0.1) / b\} .
$$

Then $A \biguplus B=\{(0.7,0.4,0.4,0.2) / a,(0.2,0.1,0.05) / b\}$.

Proposition 8. Let $(H, \circ)$ be a hypergroup and $A, B$ be fuzzy multisets of $H$. If $A$ and $B$ are fuzzy multi-hypergroups of $H$ and $A$ or $B$ is constant then $A \biguplus B$ is a fuzzy multi-hypergroup of $H$.

Proof. Without loss of generality, let $B$ be the constant fuzzy multiset of $H$ with $C M_{B}(x)=\alpha$ for all $x \in H$. We prove that the conditions of Definition 7 are satisfied for $A \biguplus B$. (1) Let $x, y \in H$ and $z \in x \circ y$. Having $C M_{A}(z) \geq C M_{A}(x) \wedge C M_{A}(y)$ implies that $\frac{C M_{A}(z)+\alpha}{2} \geq \frac{\left(C M_{A}(x) \wedge C M_{A}(y)\right)+\alpha}{2}$. We get that $C M(z)=\frac{C M_{A}(x)+\alpha}{2} \geq \frac{C M_{A}(x)+\alpha}{2} \wedge \frac{\left.C M_{A}(y)\right)+\alpha}{2}=C M(x) \wedge C M(y)$. (2) Let $a, x \in H$. Then there exist $y \in H$ such that $x \in a \circ y$ and $C M_{A}(y) \geq C M_{A}(x) \wedge C M_{A}(a)$. The latter implies that $C M(y)=\frac{C M_{A}(y)+\alpha}{2} \geq \frac{\left(C M_{A}(x) \wedge C M_{A}(a)\right)+\alpha}{2}=\frac{C M_{A}(x)+\alpha}{2} \wedge \frac{\left.C M_{A}(a)\right)+\alpha}{2}=C M(x) \wedge C M(a)$. (3) is done in a similar way to (2).

Example 15. In Example 13, $A \biguplus B=\{(0.45,0.075,0.025) / a,(0.6,0.225,0.225) / b$ is a fuzzy multi-hypergroup of $\mathrm{H}$.

Definition 11. Let $H$ be a non-empty set and $A$ be a fuzzy multiset of $H$. We define $A^{\prime}$, the complement of $A$, to be the fuzzy multiset defined as: For all $x \in H$,

$$
C M_{A^{\prime}}(x)=\left(1-\mu_{A}^{p}(x), \ldots, 1-\mu_{A}^{1}(x)\right) \text { when } C M_{A}(x)=\left(\mu_{A}^{1}(x), \mu_{A}^{2}(x), \ldots, \mu_{A}^{p}(x)\right) .
$$


Remark 4. Let $(H, \circ)$ be a hypergroup and $A$ be the constant fuzzy multi-hypergroup of $H$ defiend in Example 12. Then, $A^{\prime}$ is also a fuzzy multi-hypergroup of $H$.

We present an example of a (non-constant) fuzzy multiset $A$ of $H$ where $A$ and $A^{\prime}$ are both fuzzy multi-hypergroups of $H$.

Example 16. Let $H=\{a, b\},(H, \circ)$ be the biset hypergroup on $H$ and $A$ be a fuzzy multiset of $H$ defined as: $A=\{(0.4,0.3,0.3) / a,(0.2,0.1) / b\}$. Proposition 2 asserts that $A$ and $A^{\prime}=\{(0.7,0.7,0.6) / a,(0.9,0.8) / b\}$ are fuzzy multi-hypergroups of $H$.

Remark 5. Let $(H, \circ)$ be a hypergroup and $A$ be a fuzzy multi-hypergroup of $H$. Then, $A^{\prime}$ is not necessary a fuzzy multi-hypergroup of $H$.

The following example is an illustration for Remark 5.

Example 17. Let $(H, O)$ be the hypergroup defined in Example 9 and $A$ be the fuzzy multi-hypergroup of $H$ defined as: $A=\{(0.2,0.1) / a,(0.5,0.4,0.4) / b\}$. Then $A^{\prime}=\{(0.9,0.8) / a,(0.6,0.6,0.5) / b\}$ is not a fuzzy multi-hypergroup of $H$ as $b \in a \circ$ a and $C M_{A^{\prime}}(b) \nsupseteq C M_{A^{\prime}}(a)$.

Definition 12. Let $X$ be any set, $S \subseteq X$ and $A$ a fuzzy multiset of $X$ with fuzzy count function $C M_{A}$. Then the selection operation $\otimes$ is defined by the fuzzy multiset $A \otimes S$ with the fuzzy count function $C M$ as follows:

$$
C M(x)= \begin{cases}C M_{A}(x) & \text { if } x \in S \\ 0 & \text { otherwise. }\end{cases}
$$

Proposition 9. Let $(H, \circ)$ be a hypergroup and $S$ be a subhypergroup of $H$. If $A$ is a fuzzy multi-hypergroup of $S$ then $A \otimes S$ is a fuzzy multi-hypergroup of $H$.

Proof. Let $z \in x \circ y$. If $x \notin S$ or $y \notin S$ then $C M(z) \geq 0=C M(x) \wedge C M(y)$. If $x, y \in S$ then $z \in x \circ y$. Having $A$ a fuzzy multi-hypergroup of $S$ implies that $C M_{A}(z) \geq C M_{A}(x) \wedge C M_{A}(y)$. The latter implies that $C M(z)=C M_{A}(z) \geq C M_{A}(x) \wedge C M_{A}(y)=C M(x) \wedge C M(y)$. We prove condition 2. of Definition 7 and condition 3 is done similarly. Let $a, x \in H$. If $a \notin S$ or $x \notin S$ then for all $y \in H$ with $x \in a \circ y, C M(y) \geq 0=C M(x) \wedge C M(a)$. If $a, x \in S$ then there exist $y \in S$ such that $x \in a \circ y$ and $C M_{A}(y) \geq C M_{A}(x) \wedge C M_{A}(a)$. The latter implies that $C M(y) \geq C M(x) \wedge C M(a)$.

Definition 13. Let $X$ be any set, $S \subseteq X$ and $A$ a fuzzy multiset of $X$ with fuzzy count function $C M_{A}$. Then, the selection operation $\odot$ is defined by the fuzzy multiset $A \otimes S$ with the fuzzy count function $C M$.

$$
C M(x)= \begin{cases}C M_{A}(x) & \text { if } x \notin S \\ 0 & \text { otherwise. }\end{cases}
$$

Proposition 10. Let $(H, \circ)$ be a hypergroup and $S \subset H$ with $H-S$ a subhypergroup of $H$. If $A$ is a fuzzy multi-hypergroup of $H-S$ then $A \odot S$ is a fuzzy multi-hypergroup of $H$.

Proof. $A \odot S$ is given by the fuzzy count function $C M$ where

$$
C M(x)=\left\{\begin{array}{ll}
0 & \text { if } x \in S \\
C M_{A}(x) & \text { otherwise. }
\end{array}= \begin{cases}0 & \text { if } x \notin H-S \\
C M_{A}(x) & \text { otherwise. }\end{cases}\right.
$$

One can easily see that $A \odot S=A \otimes(H-S)$. Proposition 9 completes the proof. 
Proposition 11. $\left(H_{1}, \circ_{1}\right),\left(H_{2}, o_{2}\right)$ be hypergroups with fuzzy multisets $A, B$ respectively. If $A$ and $B$ are fuzzy multi-hypergroups of $H_{1}$ and $H_{2}$ respectively then $A \times B$ is a fuzzy multi-hypergroup of the productional hypergrpup $\left(H_{1} \times H_{2}, 0\right)$, where for all $(x, y) \in H_{1} \times H_{2}, C M_{A \times B}(x, y)=C M_{A}(x) \wedge C M_{B}(y)$.

Proof. Let $\left(x_{3}, y_{3}\right) \in\left(x_{1}, y_{1}\right) \circ\left(x_{2}, y_{2}\right)$. Then $x_{3} \in x_{1} \circ x_{2}$ and $y_{3} \in y_{1} \circ y_{2}$. Having $A, B$ fuzzy multi-hypergroups of $H_{1}, H_{2}$ respectively implies that $C M_{A}\left(x_{3}\right) \geq C M_{A}\left(x_{1}\right) \wedge C M_{A}\left(x_{2}\right)$ and $C M_{B}\left(y_{3}\right) \geq C M_{B}\left(y_{1}\right) \wedge C M_{B}\left(y_{2}\right)$. We get now

$$
C M_{A \times B}\left(x_{3}, y_{3}\right)=C M_{A}\left(x_{3}\right) \wedge C M_{B}\left(y_{3}\right) \geq C M_{A}\left(x_{1}\right) \wedge C M_{A}\left(x_{2}\right) \wedge C M_{B}\left(y_{1}\right) \wedge C M_{B}\left(y_{2}\right) .
$$

The latter implies that $C M_{A \times B}\left(x_{3}, y_{3}\right) \geq C M_{A \times B}\left(x_{1}, y_{1}\right) \wedge C M_{A \times B}\left(x_{2}, y_{2}\right)$. We now prove condition 2. of Definition 7 and condition 3. is done similarly. Let $(x, y),(a, b) \in H_{1} \times H_{2}$. Having $x, a \in H_{1}, y, b \in H_{2}$ implies that there exist $z \in H_{1}, w \in H_{2}$ such that $x \in a \circ z, y \in b \circ w$ and $C M_{A}(z) \geq C M_{A}(x) \wedge C M_{A}(a), C M_{B}(w) \geq C M_{B}(y) \wedge C M_{B}(b)$. We get that $C M_{A \times B}(z, w)=$ $C M_{A}(z) \wedge C M_{B}(w) \geq C M_{A}(x) \wedge C M_{A}(a) \wedge C M_{B}(w) \geq C M_{B}(y) \wedge C M_{B}(b)=C M_{A \times B}(x, y) \wedge$ $C M_{A \times B}(a, b)$. The latter implies that there exist $(z, w) \in H_{1}, H_{2}$ such that $(x, y) \in(a, b) \circ(z, w)$ and $C M_{A \times B}(z, w) \geq C M_{A \times B}(x, y) \wedge C M_{A \times B}(a, b)$.

Corollary 1. $\left(H_{i}, \circ_{i}\right)$ be a hypergroup with fuzzy multiset $A_{i}$ for $i=1, \ldots, n$. If $A_{i}$ is a fuzzy multi-hypergroup of $H_{i}$ then $A_{1} \times \ldots \times A_{n}$ is a fuzzy multi-hypergroup of the productional hypergroup $\left(H_{1} \times \ldots \times H_{n}, 0\right)$, where for all $\left(x_{1}, \ldots, x_{n}\right) \in H_{1} \times \ldots \times H_{n}, C M_{A_{1} \times \ldots \times A_{n}}\left(x_{1}, \ldots, x_{n}\right)=C M_{A_{1}}\left(x_{1}\right) \wedge \ldots \wedge C M_{A_{n}}\left(x_{n}\right)$.

Proof. The proof follows by using mathematical induction and Proposition 11.

Example 18. Let $(H, \circ)$ be the hypergroup defined in Example 9 and $(J, \star)$ be the biset hypergroup on the set $\{c, d\}$. Then, the productional hypergroup $(H \times J, \bullet)$ is given by the following table:

\begin{tabular}{|c|c|c|c|c|}
\hline$\bullet$ & $(a, c)$ & $(a, d)$ & $(b, c)$ & $(b, d)$ \\
\hline$(a, c)$ & $(a, c)$ & $\{(a, c),(a, d)\}$ & $\{(a, c),(b, c)\}$ & $H \times J$ \\
\hline$(a, d)$ & $\{(a, c),(a, d)\}$ & $(a, d)$ & $H \times J$ & $\{(a, d),(b, d)\}$ \\
\hline$(b, c)$ & $\{(a, c),(b, c)$ & $H \times J$ & $(b, c)$ & $\{(b, c),(b, d)\}$ \\
\hline$(b, d)$ & $H \times J$ & $\{(a, d),(b, d)\}$ & $\{(b, c),(b, d)\}$ & $(b, d)$ \\
\hline
\end{tabular}

Since $A=\{(0.2,0.1) / a,(0.5,0.4,0.4) / b\}$ is a fuzzy multi-hypergroup of $H$ and $B=$ $\{(0.3,0.05) / c,(0.2,0.2,0.1) / d\}$ is a fuzzy multi-hypergroup of $J$. Then

$$
A \times B=\{(0.2,0.05) /(a, c),(0.2,0.1) /(a, d),(0.3,0.05) /(b, c),(0.2,0.2,0.1) /(b, d)\}
$$

is a fuzzy multi-hypergroup of $H \times J$.

The following propositions (Propositions 12 and 13) deal with the strong homomorphic image and pre-image of a fuzzy multi-hypergroup.

Proposition 12. Let $\left(H_{1}, \circ_{1}\right),\left(H_{2}, O_{2}\right)$ be hypergroups, $A, B$ be fuzzy multisets of $H_{1}, H_{2}$ respectively and $f: H_{1} \rightarrow H_{2}$ be a strong homomorphism. If $A$ is a fuzzy multi-hypergroup of $H_{1}$ then $f(A)$ is a fuzzy multi-hypergroup of $\mathrm{H}_{2}$,

Proof. Let $y_{1}, y_{2} \in H_{2}$ and $y_{3} \in y_{1} \circ_{2} y_{2}$. If $f^{-1}\left(y_{1}\right)=\varnothing$ or $f^{-1}\left(y_{2}\right)=\varnothing$ then $C M_{f(A)}\left(y_{1}\right)=0$ or $C M_{f(A)}\left(y_{2}\right)=0$. We get that $C M_{f(A)}\left(y_{3}\right) \geq 0=C M_{f(A)}\left(y_{1}\right) \wedge C M_{f(A)}\left(y_{2}\right)$. If $f^{-1}\left(y_{1}\right) \neq \varnothing$ and $f^{-1}\left(y_{2}\right) \neq \varnothing$ then there exist $x_{1}, x_{2} \in H_{1}$ such that $C M_{A}\left(x_{1}\right)=\bigvee_{f(x)=y_{1}} C M_{A}(x)$ and $C M_{A}\left(x_{2}\right)=$ 
$\bigvee_{f(x)=y_{2}} C M_{A}(x)$. Having $f$ a homomorphism implies that $y_{3} \in f\left(x_{1}\right) \circ_{2} f\left(x_{2}\right)=f\left(x_{1} \circ_{1} x_{2}\right)$. The latter implies that there exists $x_{3} \in x_{1} \circ x_{2}$ such that $y_{3}=f\left(x_{3}\right)$. Since $A$ is a fuzzy multi-hypergroup of $H_{1}$, it follows that $C M_{f(A)}\left(y_{3}\right) \geq C M_{A}\left(x_{3}\right) \geq C M_{A}\left(x_{1}\right) \wedge C M_{A}\left(x_{2}\right)=C M_{f(A)}\left(y_{1}\right) \wedge C M_{f(A)}\left(y_{2}\right)$. We prove now condition 2. of Definition 7 and condition 3. is done similarly. Let $y, b \in H_{2}$. If $f^{-1}(y)=\varnothing$ or $f^{-1}(b)=\varnothing$ then for all $z \in H_{2}$ such that $y \in b \circ_{2} z, C M_{f(A)}(z) \geq 0=C M_{f(A)}(y) \wedge C M_{f(A)}(b)$. If $f^{-1}(y) \neq \varnothing$ and $f^{-1}(b) \neq \varnothing$ then there exist $x_{1}, a \in H_{1}$ such that $C M_{A}\left(x_{1}\right)=\bigvee_{f(x)=y} C M_{A}(x)$ and $C M_{A}(a)=\bigvee_{f(x)=b} C M_{A}(x)$. Having $A$ a fuzzy multi-hypergroup of $H_{1}$ implies that there exist $x_{2} \in H$ with $x_{1} \in x_{2} \circ_{1} a$ and $C M_{A}\left(x_{2}\right) \geq C M_{A}\left(x_{1}\right) \wedge C M_{A}(a)$. Since $f$ is a strong homomorphism, it follows that $y=f\left(x_{1}\right) \in f\left(x_{2}\right) \circ_{2} b$ and $C M_{f(A)}\left(f\left(x_{2}\right)\right) \geq C M_{A}\left(x_{2}\right) \geq C M_{A}\left(x_{1}\right) \wedge C M_{A}(a)=$ $C M_{f(A)}(y) \wedge C M_{f(A)}(b)$.

We can use Proposition 12 to prove Proposition 9.

Corollary 2. Let $(H, \circ)$ be a hypergroup and $S$ be a subhypergroup of $H$. If $A$ is a fuzzy multi-hypergroup of $S$ then $A \otimes S$ is a fuzzy multi-hypergroup of $H$.

Proof. Let $f: S \rightarrow H$ be the inclusion map defined by $f(x)=x$ for all $x \in S$. One can easily see that $C M_{f(A)}$ is the fuzzy count function of $A \otimes S$.

Proposition 13. Let $\left(H_{1}, \circ_{1}\right),\left(H_{2}, \circ_{2}\right)$ be hypergroups, $A, B$ be fuzzy multisets of $H_{1}, H_{2}$ respectively and $f: H_{1} \rightarrow H_{2}$ be a surjective strong homomorphism. If $B$ is a fuzzy multi-hypergroup of $H_{2}$ then $f^{-1}(B)$ is a fuzzy multi-hypergroup of $H_{1}$.

Proof. Let $x_{1}, x_{2} \in H_{1}$ and $x_{3} \in x_{1} \circ_{1} x_{2}$. Then $C M_{f^{-1}(B)}\left(x_{3}\right)=C M_{B}\left(f\left(x_{3}\right)\right)$. Having $f\left(x_{3}\right) \in f\left(x_{1} \circ_{1}\right.$ $\left.x_{2}\right)=f\left(x_{1}\right) \circ f\left(x_{2}\right)$ implies that $C M_{f^{-1}(B)}\left(x_{3}\right)=C M_{B}\left(f\left(x_{3}\right)\right) \geq C M_{B}\left(f\left(x_{1}\right)\right) \wedge C M_{B}\left(f\left(x_{2}\right)\right)=$ $C M_{f^{-1}(B)}\left(x_{1}\right) \wedge C M_{f^{-1}(B)}\left(x_{2}\right)$. We prove now condition 2. of Definition 7 and condition 3. is done similarly. Let $x, a \in H_{1}$. Having $y=f(x), b=f(a) \in H_{2}$ and $B$ a fuzzy multi-hypergroup of $H_{2}$ implies that there exist $z \in H_{2}$ such that $y \in b \circ_{2} z$ and $C M_{B}(z) \geq C M_{B}(y) \wedge C M_{B}(b)$. Since $f$ is a surjective strong homomorphism, it follows that there exist $w \in H_{1}$ such that $f(w)=z$ and $x \in z \circ_{1} w$. We get now that $C M_{f^{-1}(B)}(z)=C M_{B}(z) \geq C M_{B}(y) \wedge C M_{B}(b)=C M_{f^{-1}(B)}(x) \wedge C M_{f^{-1}(B)}(w)$.

\section{Conclusions}

In this paper, a new link between algebraic hyperstructures and fuzzy multisets was initiated and as a result fuzzy multi-hypergroups were defined and studied. In particular, different operations on fuzzy multi-hypergroups were defined and studied and several results and examples were obtained. The foundations that we made through this paper can be used to get an insight into other types of hyperstructures. As a result, different real life problems involving the concept of the fuzzy multiset can be dealt with from a different perspective.

Author Contributions: Conceptualization, S.H.-M., M.A.T. and B.D.; methodology, S.H.-M., M.A.T. and B.D.; investigation, S.H.-M., M.A.T. and B.D.; resources, S.H.-M., M.A.T. and B.D.; writing-original draft, S.H.-M., M.A.T. and B.D.; writing-review and editing, S.H.-M., M.A.T. and B.D.; project administration, S.H.-M.; funding acquisition, S.H.-M. All authors have read and agreed to the published version of the manuscript.

Funding: The APC was funded by by the Ministry of Defence in the Czech Republic.

Acknowledgments: The work presented in this paper was supported within the project for development of basic and applied research developed in the long term by the departments of theoretical and applied bases FMT supported by the Ministry of Defence in the Czech Republic.

Conflicts of Interest: The authors declare no conflict of interest. 


\section{References}

1. Marty, F. Sur une generalization de la notion de group. In Proceedings of the 8th Congress on Mathmatics Scandenaves, Stockholm, Sweden, 14-18 August 1934; pp. 45-49.

2. Antampoufis, N.; Hoskova-Mayerova, S. A Brief Survey on the two Different Approaches of Fundamental Equivalence Relations on Hyperstructures. Ratio Mathematica 2017, 33, 47-60. [CrossRef]

3. Corsini, P. Prolegomena of Hypergroup Theory, 2nd ed.; Udine: Tricesimo, Italy, 1993.

4. Vougiouklis, T. Hyperstructures and Their Representations; Hadronic Press Monographs: Palm Harbor, FL, USA, 1994.

5. Corsini, P.; Leoreanu, V. Applications of Hyperstructures Theory; Advances in Mathematics, Kluwer Academic Publisher: Dordrecht, The Netherlands, 2003.

6. Davvaz, B.; Leoreanu-Fotea, V. Hyperring Theory and Applications; International Academic Press: Cambridge, MA, USA, 2007.

7. Davvaz, B.; Cristea, I. Fuzzy Algebraic Hyperstructures; Studies in Fuzziness and Soft Computing 321; Springer International Publishing: Cham, Switzerland, 2015. [CrossRef]

8. Yager, R. On the theory of bags. Int. J. Gen. Syst. 1987, 13, 23-37. [CrossRef]

9. Onasanya, B.O.; Hoskova-Mayerova, S. Results on Functions on Dedekind Multisets. Symmetry 2019, 11, 1125. [CrossRef]

10. Zadeh, L.A. Fuzzy sets. Inform. Control 1965, 8, 338-353. [CrossRef]

11. Mei, Y.; Peng, J.; Yang, J. Convex aggregation operators and their applications to multi-hesitant fuzzy multi-criteria decision-making. Information 2018, 9, 207. [CrossRef]

12. Joshi, D.K.; Beg, I.; Kumar, S. Hesitant probabilistic fuzzy linguistic sets with applications in multi-criteria group decision making problems. Mathematics 2018, 6, 47. [CrossRef]

13. Yaqoob, N.; Gulistan, M.; Kadry, S.; Wahab, H.A. Complex intuitionistic fuzzy graphs with application in cellular network provider companies. Mathematics 2019, 7, 35. [CrossRef]

14. Miyamoto, S. Fuzzy Multisets and Their Generalizations, Multiset Processing; Springer: Berlin, Germany, 2001; pp. 225-235.

15. Onasanya, B.O.; Hoskova-Mayerova, S. Some Topological and Algebraic Properties of alpha-level Subsets' Topology of a Fuzzy Subset. Analele St. Univ. Ovidius Constanta 2018, 26, 213-227. [CrossRef]

16. Onasanya, B.O.; Hoskova-Mayerova, S. Multi-fuzzy group induced by multisets. Ital. J. Pure Appl. Math. 2019, 41, 597-604.

17. Al Tahan, M.; Hoskova-Mayerova, S.; Davvaz, B. Fuzzy multi-polygroups. J. Intell. Fuzzy Syst. 2019. [CrossRef]

18. Al Tahan, M.; Hoskova-Mayerova, S.; Davvaz, B. Some results on (generalized) fuzzy multi- $H_{v}$-ideals of $H_{v}$-rings. Symmetry 2019, 11, 1376. [CrossRef]

19. Davvaz, B. Fuzzy $H_{v}$-groups. Fuzzy Sets Syst. 1999, 101, 191-195. [CrossRef]

20. Cristea, I. Several aspects on the hypergroups associated with n-ary relations. Analele Stiint. Univ. Ovidius Constanta Ser. Mat. 2009, 17, 99-110.

21. Yaqoob, N.; Cristea, I.; Gulistan, M. Left almost polygroups. Ital. J. Pure Appl. Math. 2018, 39, 465-474.

22. Jena, S.P.; Ghosh, S.K.; Tripathi, B.K. On theory of bags and lists. Inform. Sci. 2011, 132, 241-254. [CrossRef]

23. Shinoj, T.K.; John, S.J. Intutionistic fuzzy multisets. Int. J. Eng. Sci. Innov. Technol. (IJESIT) 2013, 2, 1-24.

24. Syropoulos, A. Mathematics of Multisets, Multiset Processing; Springer: Berlin, Germany, 2001; pp. 347-358.

25. Shinoj, T.K.; Baby, A.; John, S.J. On some algebraic structures of fuzzy multisets. Ann. Fuzzy Math. Inform. 2015, 9, 77-90.

26. Davvaz, B. Polygroup Theory and Related Systems; World Scientific Publishing Co. Pte. Ltd.: Hackensack, NJ, USA, 2013.

27. Davvaz, B. Semihypergroup Theory; Elsevier/Academic Press: London, UK, 2016.

28. Leoreanu-Fotea, V.; Rosenberg, I.; Davvaz, B.; Vougiouklis, T. A new class of n-ary hyperoperations. Eur. J. Comb. 2015, 44, 265-273. [CrossRef]

29. Cristea, I. Fuzzy Subhypergroups Degree. J. Mult. Valued Log. Soft Comput. 2016, 27, 75-88.

30. Cristea, I.; Hoskova, S. Fuzzy pseudotopological hypergroupoids. Iran. J. Fuzzy Syst. 2009, 6, 11-19. 
31. Vougiouklis, T. On the hyperstructure theory. Southeast Asian Bull. Math. 2016, 40, 603-620.

32. De Salvo, M.; Fasino, D.; Freni, D.; Faro, G.L. On hypergroups with a $\beta$-class of finite height. Symmetry 2020, 12, 168. [CrossRef] 